\title{
Nose to brain delivery of drugs for CNS diseases
}

\author{
Zahraa Hussein Ali ${ }^{1 *}$, Myasar Alkotaji ${ }^{1,2}$ \\ College of pharmacy, University of Mosul, Mosul, Iraq \\ College of pharmacy, Ninevah University, Mosul, Iraq \\ Corresponding author: zahraa.php5@student.uomosul.edu.iq
}

$\frac{\text { Received }}{18-07-2021} \quad \frac{\text { Accepted }}{16-08-2021}$

\begin{abstract}
The management of central nervous system diseases is extremely challenging due to the numerous obstacles that stand against the successful delivery of drugs to their target site in the brain. Defeating the blood-brain barrier is considered the most significant challenge in this delivery. Different alternative routes of administration have been investigated. Nasal delivery is one of the possible ways for direct brain targeting. The nasal mucosa is the only part of the body at which the external environment become in straight connection with the central nervous system which takes place through the olfactory portion of the nasal mucosa. Different mechanisms have been suggested to describe the pathway for straight nasal to brain transport of medications, however, the precise route is still vague. The most important proposed pathways include nerve pathways (olfactory and trigeminal nerve), vascular, lymphatic, and cerebrospinal fluid mediated pathways. Among these mechanisms, the olfactory intra-neuronal delivery is the dominant one. Various particulate systems have been investigated for nasal delivery with the intention of direct transport to the brain. The most frequently studied delivery systems are polymeric nanosystems, lipid based nanosystems, and nanometric emulsions. In conclusion, direct nasal-to-brain delivery is a very fertile research area and any achievements in this area are running side by side with the progressions that occur in the particulate systems.
\end{abstract}

Keywords: Nasal to brain delivery, Nasal delivery, particulates system, CNS targeting

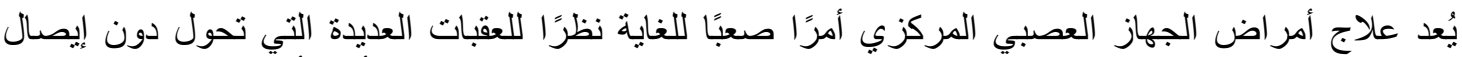

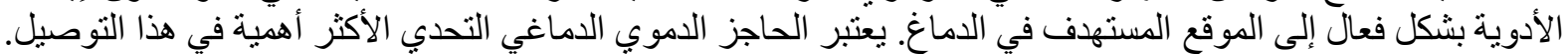

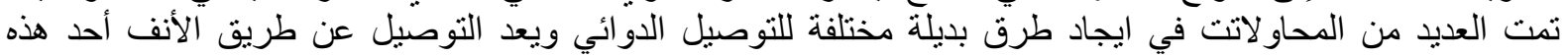

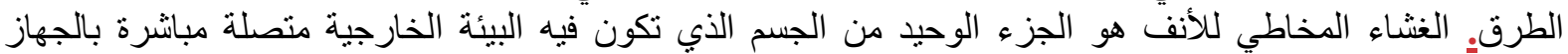

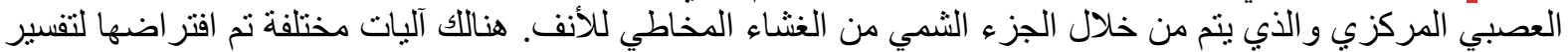

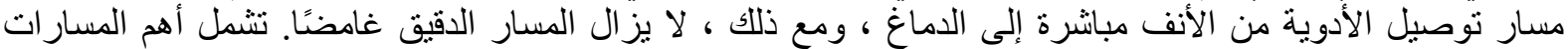

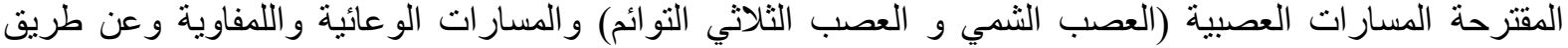
مسار السائل النخاعي. من بين هذه الآليات ، يعتبر التوصيل الثمي داخل الخلايا العصبية هو السائد.

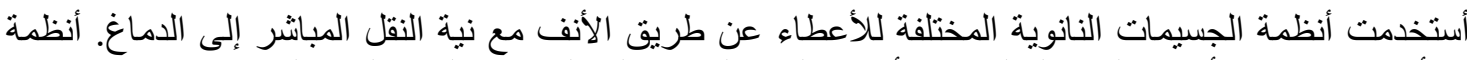
التوصيل الأكثر در اسة هي أنظمة النانو البوليمرية وأنظمة النانو القائمة على الدهون والفية المستحلبات النانومثرية.

في الختام ، يعتبر نوصيل الأنف المباشر إلى الدماغ مجال بحث خصب للغاية وأي إنجاز ات في هذا المجال تأتي جنبًا إلى جنب مع التقدم الذي يحدث في أنظمة الجسيمات. 
الكلمات المفتاحية: نوصيل الانف الى الدماغ، توصيل الانف، نظام الجسيمات، استهداف الجهاز العصبي المركزي.

\section{Introduction}

Central nervous system (CNS) diseases are a collection of diseases (including epilepsy, Alzheimer's disease, schizophrenia, Parkinson disease, brain tumors, and others) that affect the central nervous systems causing a plethora of symptoms that interfere with the mental or physical status of an individual (1). In spite of the great progress in the generation of drug delivery systems for the treatment of CNS disorders, the effective treatment of these diseases is still very challenging (1). Numerous obstacles are present that need to be overcome by CNS medications in order to reach their active site in the brain. The principal one is the presence of the blood-brain barrier that coats the microvasculature of the brain acting as a fence separating the systemic bloodstream from the tissues of the CNS (2). The BBB is designated by the overly condensed endothelial cells that extremely influence the paracellular movement of molecules. This tightly packed cellular layer would preserve the brain from hurtful substances like toxins, microorganisms, heavy metals, and others but at the same time restrict the passage of therapeutic agents to the brain (1)(2). In addition, there are many efflux transporters distributed along with the BBB. These transporters are highly nonselective and have the ability to actively expel wide diversity of foreign molecules which have successfully entered the brain back to the systemic circulation once again (2)(3). Many CNS medications are substrates of these efflux transporters which would further compromise their ability to reach to their target in effective concentrations (2)(3).

Nowadays, most CNS drugs are administered orally and, to a lesser extent, parenterally (4). However, these administration routes are linked to many disadvantages (4). Firstly, the oral rout is susceptible to first-pass metabolism in the liver and linked to elevated incidence of peripheral side effects (4). Also, it is inappropriate for patients suffering from swallowing difficulty or vomiting. On the other hand, the parenteral route is invasive and associated with poor patient compliance (4). Accordingly, there is increased effort to develop formulations of CNS medications that are suitable for administration through alternative routes (4).

\section{Nose as alternative administration route for CNS drugs}

The nasal route for drug administration is one of the most broadly explored routes for the effective delivery of CNS medications. It has numerous advantages that make it an attractive route of administration including the highly permeable mucosa, abundant blood flow, and the bypassing of the first metabolism effect in the liver since the venous blood return directly to the heart without passing, first, through the liver (5)(6). In addition, multiple published researches specified a lower occurrence of obesity when conventional tablets of olanzapine, for example, are exchanged by oral dispersible dosage forms or following switching to injectable formulations. These findings suggested a positive correlation between oral intake of conventional tablets and metabolic side effects (7)(8).

Furthermore, there is a great likelihood for the drugs administered intranasally to pass directly to the brain (9). This route is brought about by the existence of olfactory neurons within the olfactory region of the nasal mucosa. The axons of these neurons are grouped together forming nerve fibers that traverse the blood-brain barrier (BBB) and enter the brain. This fact is extremely important 
since it provides a chance for direct noseto-brain delivery and circumventing the BBB (9)(10).

The harsh nasal environment with the rapid mucociliary clearance are the most important challenges in the nasal delivery of medications (11)(12). Different strategies and techniques have been investigated to overcome these limitations and prolong the drug contact time with the absorbing epithelium leading to higher nasal bioavailability. For instance, different particulate systems (like nano and micro emulsions/suspensions, liposomal systems, and solid nanoparticles) have been investigated to achieve better protection of the drug molecule along with the improved nasal permeation leading to increased systemic bioavailability (13)(14).

\section{Nose anatomy and physiology}

The nasal cavity begins anteriorly at the nostrils and extends posteriorly until it becomes communicated with the rhinopharynx (15). The nasal cavity is divided into two identical parts by a septum which comprises bony, and cartilaginous portions (15)(16). Each half is made of three zones: the nasal vestibule, the respiratory zone and the olfactory zone with an overall surface area of the nasal cavity of around $1.5 \mathrm{~m}^{2}$ (16)(17). All the regions of the nasal mucosa are expressed in figure (1).

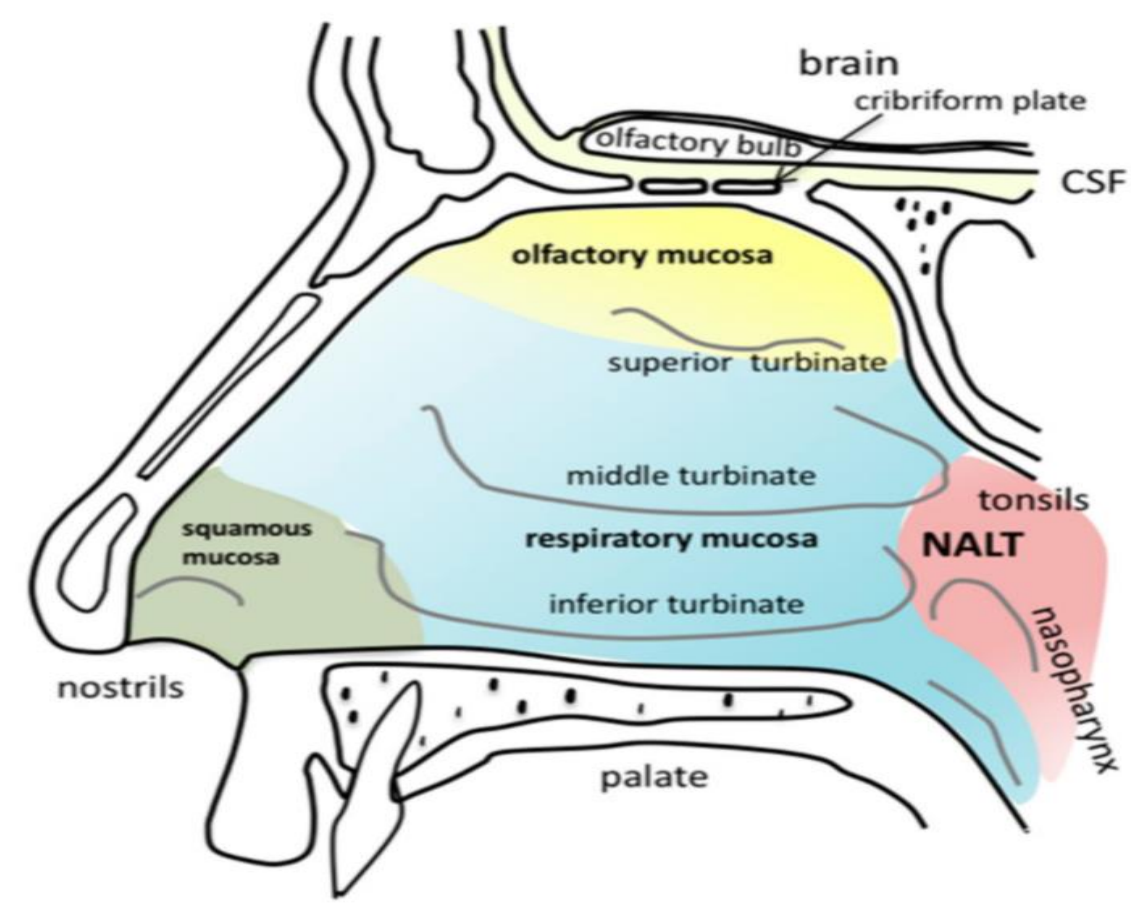

Figure (1): Different regions of nasal mucosa. The green area for the squamous mucosa, the blue area for the respiratory mucosa, and the yellow area for the olfactory mucosa (18).

The nasal mucosa is lined by the nasal epithelial layer with a cellular composition that differs according to the location within the nasal cavity (16)(19). The front third of the nasal cavity is covered with a squamous and transitional epithelium composed of stratified epithelium that accommodates cuboidal cells along with microvilli while the back two-thirds of the nasal cavity are covered with pseudostratified columnar epithelium (respiratory epithelium) in which four types of cells exist: ciliated, non-ciliated, goblet and basal cells (15)(16)(19).

The ciliated cells account for about eighty percent of the cellular population in the posterior nasal mucosa $(15)(16)$. They 
are covered with cilia that are anchored to the cell through a basal body from which they are emerged (15). These ciliated cells play a major role in the mucociliary clearance activity, which serves to protect the nasal passages and play a significant part in restricting the residence time of the drugs that are administered intra-nasally (17). The goblet cells secrete acid-based mucin with a specific viscosity and elasticity that are required for good nasal clearance (16). The epithelial layer is supported on a basement membrane and lamina propria (15). The basement membrane differs from those present elsewhere in the body in that it is a highly permeable membrane due to the presence of vessels penetrating it through which fluids can directly spread onto the mucosal surface (15). The lamina propria carries all the glands, nerve endings, and vascular elements (15).

The nose can serve both respiration and olfaction functions (15)(16). In respiration, the nose acts to filter, moisturize and warm the inhaled air as it passes along the nasal passages until reaching the alveoli within the lungs. It is considered as the first defence against irritants, micro-organisms, allergic substances, and others that can enter the nasal cavity during inspiration (16).

On the other hand, olfaction is mediated by the presence of the numerous olfactory receptors that exist in the olfactory region of the nasal mucosa (16)(19). This area is restricted to a very small location including the nasal cavity roof, superior part of the lateral nasal wall, and the superior third of the nasal septum (20). It is estimated that there are 10 to 20 million receptors present in the olfactory region (20). These receptors act as peripheral sensory receptors and the axons of which are grouped together forming the olfactory nerve fiber that terminates in the olfactory bulb glomeruli (20). The olfactory area is found to play a fundamental role in the direct delivery of drugs from the nose to the brain (10).

\section{Applications of nasal administration route}

The nasal route has been recognized for the topical delivery of medications for many years (21). Antihistamines, decongestants, and corticosteroids are among the most common drugs administered intranasally with the intention to produce local effects (22). These medications are used for the alleviation of allergic symptoms that accompany seasonal rhinitis (22)(23). Also, the nasal route has been investigated for the systemic delivery of drugs used for the treatment of various conditions including pain, insomnia, vomiting, headache, hormonal replacement, prostate cancer, osteoporosis, and others (14).

Furthermore, there is growing attention to nasal route utilization for immunization (26). The nasal mucosa is regarded as an appropriate site for the stimulation of topical (mucosal) and systemic immunological responses (26)(27). Apart from the systemic responses, the nasal mucosal surfaces possess specific sites for the induction and initiation of immunological reactions in response to specific antigens. These nasal sites are referred to as the nose-associated lymphoid tissues (NALT) and they can work irrespectively of the systemic immune responses (26)(27). Many published articles revealed the safety and efficacy profile of intranasal vaccination (27)(28). Nowadays, there are two vaccines that are approved for intranasal administration (27)(28). Both of them were approved for influenza immunization in the USA and Asia and contain live attenuated viruses (27).

\section{Nasal route for the treatment of CNS diseases}

CNS diseases, such as epilepsy, schizophrenia, Alzheimer's, Parkinson, and 
others, are defined as disorders that affect different parts of the brain (29). As stated by the world health organization (WHO), millions of people are suffering from neurological diseases worldwide (29). Medications used for the treatment of these disorders are usually given orally or parenterally. In order to be effective, the CNS medications should be transferred from the systemic bloodstream into the brain (where their active target is located) by crossing the blood-brain barrier (BBB). The BBB is a membrane layer that surrounds the microvasculature of the brain creating an interface between the blood and the tissues of the CNS (2)(3). It serves as a diffusion layer that regulates the passage of nutrients, drugs as well as other chemicals from the blood to the neuronal tissues thus regulating the proper functioning of the CNS (2)(30).

Three cellular elements constitute the BBB including the endothelial cells, the astrocyte end-feet, and pericytes (2). The endothelial cells of the BBB are unique from those in other parts of the body by the presence of tight joints between cells and the scattering of pinocytic transport vesicles (2)(31). These tight junctions restrict the paracellular movement of water-soluble molecules (including many therapeutic agents) while small lipophilic molecules can diffuse transcellular across the plasma membrane in accordance with their concentration gradient (31). Furthermore, the entrance of many lipid-soluble molecules into the brain is impeded by the availability of diverse efflux transporters that belong to the ABC-binding cassette. These transporters are non-specific and have the capability to actively efflux a broad scope of highly lipid-soluble substances that could spread easily through the plasma membrane, back to the blood (30)(31). Large entities (including many nutrients) can infiltrate to the brain by approaches that are facilitated by transporters and receptor-mediated endocytosis (31). All of these obstacles increase the difficulty for the CNS drugs to reach their active sites (4)(32).

The nasal mucosa is the only place in the body at which the brain becomes in a close connection with the outer environment (3). This link is attained by the axons of nerves (olfactory and trigeminal) which pass through the nasal mucosa permitting direct contact (29). Hence, there is a great possibility for the delivery of medications administered intranasally through the olfactory nerves directly to the brain and thus circumventing the BBB (25)(29). This pathway is very promising for the delivery of CNS drugs since the BBB is the most significant limitation restricting the reach of these medications to their target site in the brain (1). Figure (2) represents the possible direct delivery route of drugs administered intranasally to the brain. 


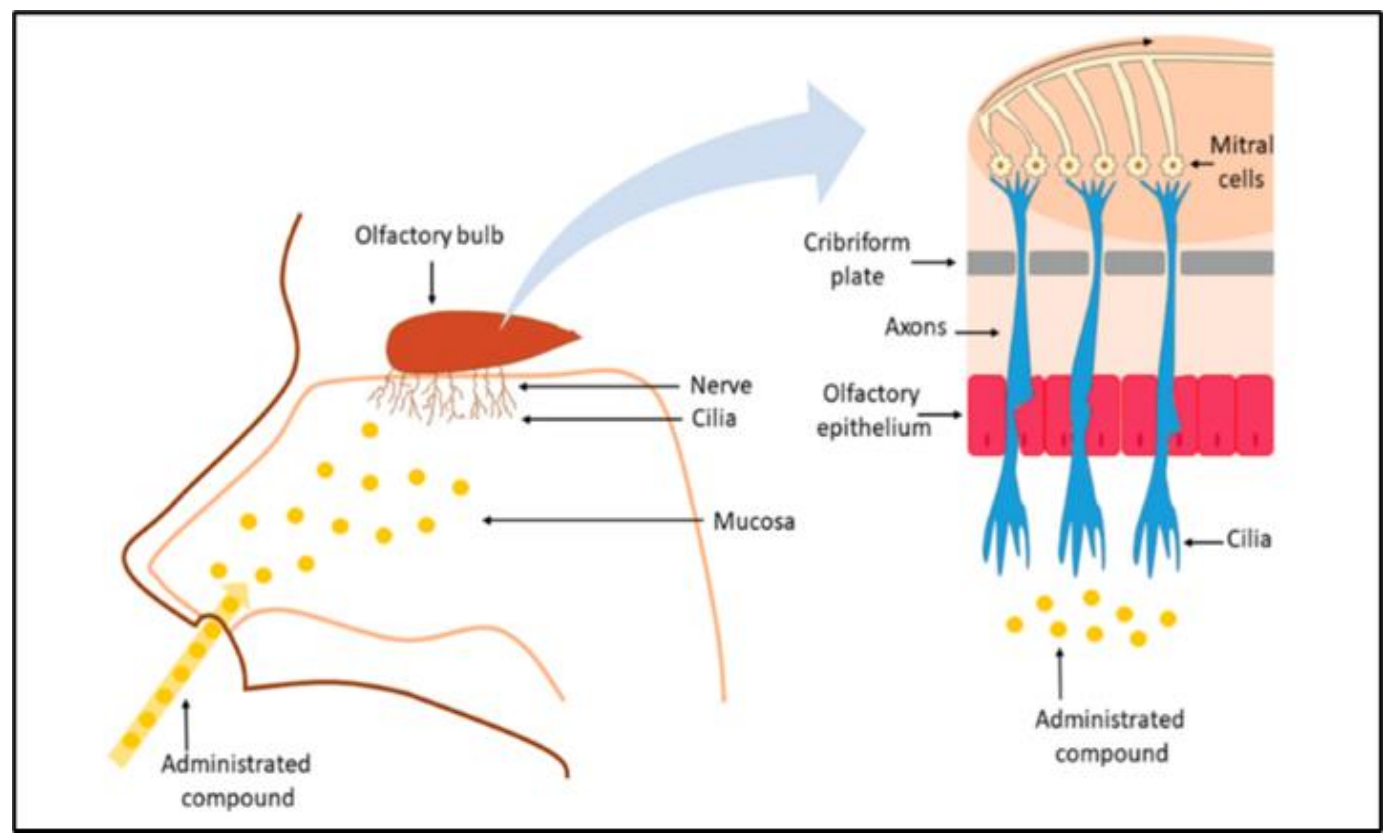

Figure (2): The possible route for direct delivery of CNS medication to the brain through nasal cavity (32).

\section{Direct nose-to brain delivery mechanisms}

The absolute mechanism by which drug molecules enter the brain from the nasal cavity is not fully understood (29). Nevertheless, it has been estimated the presence of five different delivery pathways for the transportation of the drug from the nasal cavity to the brain (32). These include the olfactory and trigeminal nerve pathways, the vascular, the lymphatic and the cerebrospinal fluid (CSF) pathways (29)(32). Depending on the drug's molecular characteristics, formulation properties, and physiological circumstances, the nose to brain delivery of the medication may happen by a single pathway or a collection of multiple pathways (32).
After nasal application, if the drug molecule is settled on the respiratory epithelium, it would be absorbed into the lymphatic or vascular systems and then enter to the CNS from the systemic circulation (29). While, if the deposition of the drug molecule takes place on the olfactory epithelium, then it would be transported to the brain through the olfactory neurons (by intercellular or intracellular mechanisms) or olfactory epithelial cells (by transcellular or paracellular mechanisms) (29)(33). The same process occurs if the drug is transmitted through the trigeminal nerve (which innervates both, the olfactory epithelium and the respiratory epithelium) (32). All the pathways for the direct delivery of the drug from the olfactory epithelium to the brain are represented in figure 


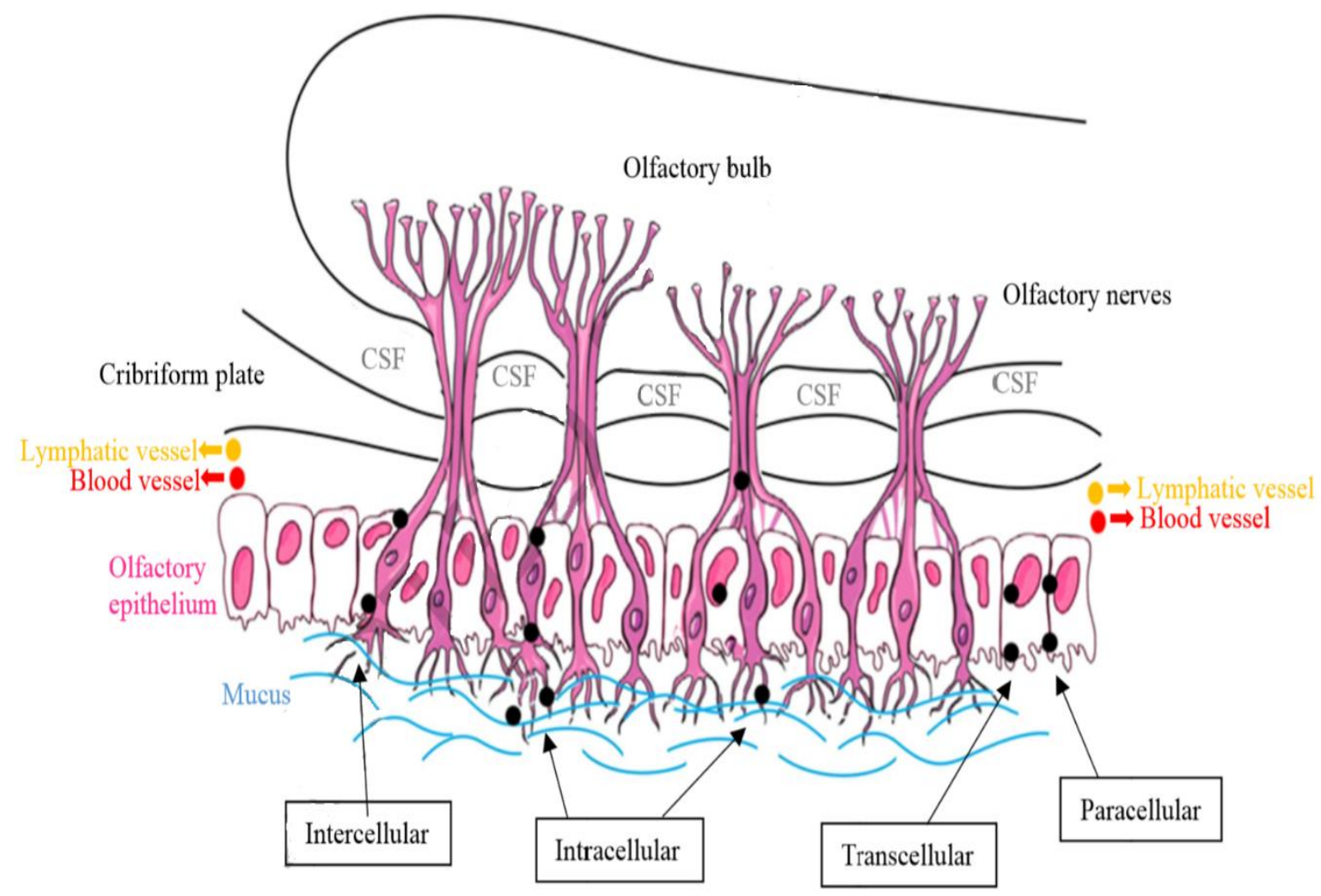

Figure (3): The different pathways for the direct delivery of the drug from the olfactory epithelium to the brain (33).

The transcellular mechanism may occur by passive diffusion, pinocytosis, or by receptor-mediated endocytosis. The paracellular mechanisms require the penetration of the drug molecule across the tight junction between the epithelial cells, while the intercellular migration involves the extracellular permeation of the active pharmaceutical ingredient along the neuronal axons (29). The paracellular and the intercellular diffusion mechanisms are brought about by the fact that the nerveregeneration of the cranial nerves (including the trigeminal and the olfactory nerves) would convert the epithelium into a very porous state which permits the rapid transfer of molecules across it (33). Finally, the intracellular transmission involves the drug molecule absorption (by pinocytosis or endocytosis) into the neuronal interior with subsequent intracellular transportation along the neuronal axons to the olfactory bulb (29)(33).

Of the above mechanisms, the olfactory intra-neuronal pathway is the primary route for the nose to brain delivery of CNS medications (particularly for the lipophilic drug molecules) (29). But, it is believed that the passage of the drug by this pathway needs from 1.5 to 6 hours with the consequence of retarded onset of action (33). However, there is a great potential for arterial pulsation to take place within the nasal cavity resulting from the high vascularization of the nasal mucosa (33). This phenomenon would shorten the time required for the drug molecule to pass through the neuronal axons to the brain into about 20 minutes (33). 


\section{Types of nano-carrier formulations used in nose-to-brain delivery}

There is a growing interest in the generation of nano-carrier-based systems for the direct nose to brain delivery of medications (34). The use of such systems is associated with an improvement in the drug absorption with elevated efficiency of the delivery making them fascinating delivery systems (34). In addition, the development of the nano-carrier systems can be adjusted with respect to the proper selection of excipients, the physicochemical characteristics of the resulting system (including the particle dimensions, surface charge, and others), drug release profile, biocompatibility, biodegradability and others (34). Polymerbased, lipid-based and nanometric emulsions have been explored extensively for intranasal delivery of active pharmaceutical ingredients (34)(35).

A

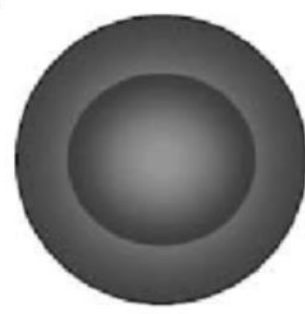

Polymeric
B

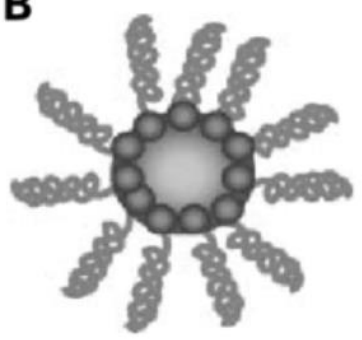

Polymeric micells

\section{Polymeric nanosystems}

These were first discovered four decades ago and they gain a huge interest in the manufacture of drug delivery systems in neurological disorders treatment (33). Based on the resulting structure of the nanosystem, they could be classified into polymeric nanoparticle (polymers at the core), polymeric micelles (with the use of amphiphilic polymers), and dendrimers (with the use of branched polymers) (33)(35)(36). Their sizes vary widely but within the nanometre scale (35). The different forms of some of the polymeric nanosystems are represented in figure (4).

Figure (4): The different forms of the polymeric nanosystems; polymeric nano particles (A), polymeric micelles (B) and dendrimers (C) (36).

Both natural (such as chitosan and alginates) and synthetic polymers (such as polyesters) could be used for the fabrication of polymeric nanosystems (34). However, due to their documented capability to obtain sustained drug release along with biocompatibility and biodegradability profiles, the FDA approved polymers of poly (lactide-coglycolic acid) known as (PLGA) and poly (lactic acid) known as (PLA). These polymers are the chief synthetic studied polymers for the entrapment of drugs intended for the nose to brain delivery (33)(34).

The polymeric nanoparticles are compacted systems that usually have a polymer matrix in which the drug molecule could be dissolved, attached, entrapped, or encapsulated (35). In contrast, polymeric micelles are selfassembled structures made of amphiphilic 
copolymers (35). They have a lipophilic core with a hydrophilic shell so they are very useful for the encapsulation of lipophilic drugs (35).

Pranav et al. (41) prepared PLGA nanoparticles loaded with lamotrigine for the treatment of epilepsy through the direct nose to brain delivery. Emulsification followed by the solvent-evaporation method was used for the fabrication of the loaded nanoparticles. Various techniques were employed to evaluate the brain delivery including pharmacodynamic and Gama scintigraphy studies in mice. The Gama scintigraphy and the biodistribution studies revealed a higher brain deposition of the medication following the intranasal intake of the lamotrigine-loaded PLGA nanoparticles as compared to lamotrigine plain solution. The pharmacodynamic study demonstrated that the onset of seizure was delayed with the nanoparticle formulation in comparison with the solution administration. These results suggested that the prepared lamotrigineloaded PLGA nanoparticles were good substitutes to the current epilepsy formulations but further evaluation in higher animals as well as in human volunteers is recommended.

PLGA nanoparticles loaded with olanzapine for the nose to brain delivery were developed by Seju et al. (45). The nanoprecipitation method was used for the production of nanoparticles. The prepared nanoparticles were evaluated by in-vitro, ex-vivo, and in-vivo experiments. The invivo pharmacokinetic study was conducted on rat animal model. Following intranasal intake of the prepared nanoparticles, the concentration of the drug in the brain was measured by HPLC. This concentration was compared with the brain concentration following intranasal intake of olanzapine solution and after intravenous intake of olanzapine PLGA nanoparticles (which were previously diluted with phosphate buffer). They found that the order of brain concentration of olanzapine was decreasing in the following order: olanzapine PLGA nanoparticles (IN) > olanzapine PLGA nanoparticles (IV) > olanzapine solution (IN). These findings clearly suggested the direct delivery of olanzapine from the nose to the brain.

\section{Lipid-based nanoparticles}

In 1960, liposomes were discovered as the earliest lipid-based nanocarriers to be invented and became approved as drug delivery systems by the FDA (33). Liposomes are spherical vesicular structures composed of an aqueous centre surrounded by either single or multiple phospholipid bilayers (34-35). They have the advantages of being biocompatible, biodegradable, and having the ability to incorporate either hydrophilic (within the core), or hydrophobic (within the shell) active pharmaceutical ingredients (APIs). Their most remarkable drawback is their rapid degradation with a very short halflife (33). This drawback had been defeated by the fabrication of proliposomes which are anhydrous granular entities that are transformed into liposomes by hydration (33).

Raju et al. (40) developed liposomes for the direct nose to brain delivery of imatinib mesylate (anti-cancer medication). In-vitro and in-vivo evaluations in the rat model were performed on the prepared liposomes to assess the brain targeting in contrast to the pure drug solution. They found that the area under the curve (AUC) for the relationship between the brain concentration of the drug versus time following the intranasal administration of the liposomal formulation was much higher than the pure drug solution. These findings confirmed the higher brain targeting of the prepared liposomes.

On the other hand, solid lipid nanoparticles (SLN) are comprised of a biocompatible central core made of solid lipids (such as triglycerides) that are 
enclosed and stabilized by different surface-active agents (35)(34). The active pharmaceutical ingredient is usually incorporated into the solid lipid core (34). Therefore, these structures are most commonly used for lipophilic medication and, to a lesser extent, for hydrophilic macromolecules (34).

For instance, a mucoadhesive in-situ gel-based solid lipid nanoparticles (SLN) loaded with almotriptan was fabricated by Nancy et al. (43). Different concentrations of Poloxamer 407 (as the in-situ gelling agent) along with different polymers (including Carbopol 974, sodium alginate, and sodium CMC as mucoadhesive agents) were investigated. The optimized formula contained $18 \%$ of Poloxamer 407 with $0.75 \%$ of sodium CMC. The brain targeting efficiency was concluded by invivo pharmacokinetic studies in rat models. Histopathological and biomarkers evaluation confirmed the fast brain delivery of almotriptan as manifested by the 10 minutes' brain/blood ratio of 0.89 , 0.19 and 0.31 for the almotriptan loaded SLN based nasal in-situ gel (IN), plain almotriptan based nasal in-situ gel (IN) and almotriptan solution (IV), respectively.

\section{Microemulsion and nanoemulsion}

Nanometric

emulsions (nanoemulsions and microemulsions) are biphasic liquid dispersions of two immiscible liquids forming either oil in water or water in oil emulsion stabilized by a suitable blend of emulsifying agents (surfactants and co-surfactants) (37)(38). There is no clear cut-off definition to distinguish between nanoemulsion and microemulsion (35). However, thermodynamic stability is good parameter that helps differentiate between these two nanometric dispersion systems (37)(38). The microemulsions are thermodynamically stable while nanoemulsions lack this property (but they have a good kinetic stability) (37)(38). Therefore, high energy is required for the fabrication of nanoemulsion in contrast to microemulsion which is self-assembled and formed spontaneously upon proper blending of oil with the aqueous phase in the presence of a suitable surfactant mixture (for stabilization) (34).

The nano-sized emulsions are the most frequently investigated drug delivery systems for the direct nose to brain targeting (35). The reason behind this is the exceptional advantages profile (35). Firstly, the small droplet size would increase the permeability and prevent the destabilization mechanisms including sedimentation, coalescence, and creaming resulting in prolonged physical stability (37). Secondly, they could be incorporated into various types of formulations like liquids, sprays, creams, ointments, gels, and others which could be delivered by the nasal route (38). Also, they offer an excellent solution for stability or solubility problems that accompany many drugs (38). For instance, hydrophobic drugs could be dissolved in the oil phase and after administration, the drug will be released from oil droplets into the surrounding aqueous environment where nanoprecipitation takes place. These extremely small-sized precipitates provide extensively large surface area with a greatly enhanced dissolution rate and improved bioavailability (38). With respect to drug stability, nanoemulsions and microemulsions can protect the drug (by encapsulating it within oil droplets) from enzymatic degradation, hydrolysis, oxidation, and other instability issues at physiological circumstances (38). Therefore, they provide a very promising dosage form for delivering lipophilic drugs intranasally. However, one of the important limitations for all liquid dosage forms for nasal delivery, including the nanomulsion, is the short residence time.

Kumar et al. (39) prepared olanzapine in a nano emulsion-based 
formulation with a mucoadhesive property for the nose to brain targeting. The olanzapine nanoemulsion was prepared by an aqueous-titration technique using oil phase (Capmul 15\% wt/wt), surfactant (Tween 80 at $35 \% \mathrm{wt} / \mathrm{wt}$ ), and cosurfactants (ethanol and PEG 400 in a (1:1) ratio at a concentration of $17.5 \%$ $\mathrm{wt} / \mathrm{wt}$ ). The olanzapine mucoadhesive nanoemulsion was made through the addition of polymer (chitosan $0.5 \% \mathrm{wt} / \mathrm{wt}$ ) to the olanzapine nanoemulsion. Gamma scintigraphy studies were performed on rats using ${ }^{99 \mathrm{~m}} \mathrm{Tc}$ labelled olanzapine. The ratios of olanzapine brain to blood distribution after 30 minutes of administration (using equal doses of the drug) exhibited a clearly enhanced accumulation of the drug in the rat's brain.

Segar et al. (42) developed microemulsion-based aqua-sensitive insitu gel of diazepam for intranasal administration with enhanced brain uptake as an urgent treatment of epilepsy and as a substitute to injections. The components of the microemulsion include Capmul MCM (as the oil phase), Tween 80 (as surfactant), and propylene glycol (as cosurfactant). The formulation did not contain any polymer and the gelling occurred rapidly in contact with a small amount of water forming a mucoadhesive gel. Brain microdialysis was performed on rats to validate the concentration of the free diazepam that is present in the brain. The results revealed a twofold increase in the potential of the direct nose to brain transmission of diazepam from the diazepam-loaded aqua-triggered in-situ gel microemulsion when compared to the diazepam solution following intranasal intake.

\section{References}

1. Khan AR, Liu M, Khan MW, Zhai G. Progress in brain targeting drug delivery system by nasal route. J Control Release [Internet].
Furthermore, thermo-sensitive nasal in-situ gel was produced by Masoumeh et al. This gel was loaded with a temozolomide nanoemulsion, and investigated for the treatment of glioblastoma through a direct nose to brain delivery (44). Based on the pseudo ternary phase diagrams, Labrasol, Transcutol, and Triacetin were selected to be the components of the nano emulsion. Poloxamer derivatives were selected to be incorporated as the in-situ gelling agent within the formulation. Gamma scintigraphy studies, using radiolabelled $\left({ }^{99 \mathrm{~m}} \mathrm{Tc}\right)$ temozolomide, were conducted on rats to assess the brain targeting of the formulation. The resulted images showed very good brain accumulation after intranasal administration.

\section{Conclusions}

Direct nose to brain delivery is a promising way for the delivery of drugs for CNS diseases. It has been manipulated for different drugs and for different diseases. The improvement in this field of drug delivery will be in parallel to the developments in the field of nanoparticulate systems, as most of the successful drug delivery systems for this application are nanoparticulated including nanoparticles, liposomes, and nanoemulsions. Although this way of drug targeting is still in its infancy stage, there will be a huge expansion in the near future.

\section{Acknowledgments}

Both researchers are grateful to the College of Pharmacy-University of Mosul.

2017;268(August):364-89.

Available from: https://doi.org/10.1016/j.jconrel.201 7.09.001

2. Ballabh P, Braun A, Nedergaard M. 
The blood-brain barrier: An overview: Structure, regulation, and clinical implications. Neurobiol Dis. 2004;16(1):1-13.

3. Ul Islam S, Shehzad A, Bilal Ahmed $\mathrm{M}$, Lee YS. Intranasal delivery of nanoformulations: A potential way of treatment for neurological disorders. Molecules. 2020;25(8):127.

4. Kaminsky BM, Bostwick JR, Guthrie SK. Alternate Routes of Administration of Antidepressant and Antipsychotic Medications. Ann Pharmacother. 2015;49(7):808-17.

5. Suresh S, Bhaskaran S. Nasal drug delivery: An overview. Indian $\mathbf{J}$ Pharm Sci. 2005;67(1):19-25.

6. Marx D, Williams G, Birkhoff M. Intranasal Drug Administration An Attractive Delivery Route for Some Drugs. Drug Discov Dev From Mol to Med. 2015;

7. Crocq MA, Guillon MS, Bailey PE, Provost D. Orally disintegrating olanzapine induces less weight gain in adolescents than standard oral tablets. Eur Psychiatry. 2007;22(7):453-4.

8. Sağlam Aykut D, Özkorumak Karagüzel E. A comparison of depot and oral atypical antipsychotics in terms of metabolic syndrome markers. Psychiatry Clin Psychopharmacol. 2018;28(3):28590.

9. Türker S, Onur E, Ózer Y. Nasal route and drug delivery systems. Pharm World Sci. 2004;26(3):13742.

10. Wang Z, Xiong G, Tsang WC, Schätzlein AG, Uchegbu IF. Noseto-brain delivery. 2008;23(13):275281.
11. Jassim ZE, Al-akkam EJ. A review on strategies for improving nasal drug delivery systems. Drug Invent Today. 2018;10(1):1-9.

12. Gänger S, Schindowski K. Tailoring formulations for intranasal nose-tobrain delivery: A review on architecture, physico-chemical characteristics and mucociliary clearance of the nasal olfactory mucosa. Pharmaceutics. 2018;10(3).

13. Erdő F, Bors LA, Farkas D, Bajza Á, Gizurarson S. Evaluation of intranasal delivery route of drug administration for brain targeting. Brain Res Bull. 2018;143(July):155-70.

14. Cunha S, Amaral MH, Sousa Lobo JM, Silva AC. Lipid nanoparticles for nasal/intranasal drug delivery. Crit Rev Ther Drug Carrier Syst. 2017;34(3):257-82.

15. Van Cauwenberge P, Sys L, De Belder T, Watelet JB. Anatomy and physiology of the nose and the paranasal sinuses. Immunol Allergy Clin North Am. 2004;24(1):1-17.

16. Watelet JB, Van Cauwenberge P. Applied anatomy and physiology of the nose and paranasal sinuses. Allergy Eur J Allergy Clin Immunol Suppl. 1999;54(57):14-25.

17. Anand U, Feridooni T, U. R. Novel Mucoadhesive Polymers for Nasal Drug Delivery. Recent Adv Nov Drug Carr Syst. 2012;

18. Jain A, Hurkat P, Jain A, Jain A, Jain A, Jain SK. Thiolated Polymers: Pharmaceutical Tool in Nasal Drug Delivery of Proteins and Peptides. Int $\mathbf{J}$ Pept Res Ther [Internet]. 2019;25(1):15-26. Available from: http://dx.doi.org/10.1007/s10989018-9704-y 
19. Chatterjee B, Gorain B, Mohananaidu K, Sengupta P, Mandal UK, Choudhury $\mathrm{H}$. Targeted drug delivery to the brain via intranasal nanoemulsion: Available proof of concept and existing challenges. Int $\mathrm{J}$ Pharm [Internet]. 2019;565(March):25868. Available from: https://doi.org/10.1016/j.ijpharm.20 19.05.032

20. Geurkink N. Nasal anatomy, physiology, and function. J Allergy Clin Immunol. 1983;72(2):123-8.

21. Suresh S, Bhaskaran S. Nasal drug delivery: An overview. Indian J Pharm Sci. 2005;67(1):19-25.

22. Keller LA, Merkel O, Popp A. Intranasal drug delivery: opportunities and toxicologic challenges during drug development. Drug Deliv Transl Res [Internet]. 2021;(0123456789). Available from: https://doi.org/10.1007/s13346-02000891-5

23. Gutiérrez-Cardona N, Sands $\mathrm{P}$, Roberts G, Lucas JS, Walker W, Salib R, et al. The acceptability and tolerability of nasal douching in children with allergic rhinitis: A systematic review. Int $\mathrm{J}$ Pediatr Otorhinolaryngol. 2017;98:126-35.

24. Verekar RR, Gurav SS, Bolmal U. Thermosensitive mucoadhesive in situ gel for intranasal delivery of Almotriptan malate: Formulation, characterization, and evaluation. $\mathbf{J}$ Drug Deliv Sci Technol [Internet]. 2020;58(December 2019):101778. Available from: https://doi.org/10.1016/j.jddst.2020. 101778

25. Selvaraj K, Gowthamarajan K, Karri VVSR. Nose to brain transport pathways an overview: potential of nanostructured lipid carriers in nose to brain targeting. Artif Cells, Nanomedicine Biotechnol [Internet]. 2018;46(8):2088-95. Available from: https://doi.org/10.1080/21691401.2 017.1420073

26. Bernocchi B, Carpentier R, Betbeder D. Nasal nanovaccines. Int J Pharm [Internet]. 2017;530(12):128-38. Available from: http://dx.doi.org/10.1016/j.ijpharm. 2017.07.012

27. Bahamondez-Canas TF, Cui Z. Intranasal immunization with dry powder vaccines. Eur J Pharm Biopharm [Internet]. 2018;122(November):167-75.

Available from: https://doi.org/10.1016/j.ejpb.2017. 11.001

28. Lobaina Mato Y. Nasal route for vaccine and drug delivery: Features and current opportunities. Int $\mathbf{J}$ Pharm [Internet]. 2019;572:118813. Available from: https://doi.org/10.1016/j.ijpharm.20 19.118813

29. Aderibigbe BA. In situ-based gels for nose to brain delivery for the treatment of neurological diseases. Pharmaceutics. 2018;10(2).

30. Banks WA. The blood brain barrier. Neuroimmune Pharmacol. 2008;2138.

31. Abbott NJ, Patabendige AAK, Dolman DEM, Yusof SR, Begley DJ. Structure and function of the blood-brain barrier. Neurobiol Dis [Internet]. 2010;37(1):13-25. Available from: http://dx.doi.org/10.1016/j.nbd.2009 .07 .030

32. Bruinsmann FA, Vaz GR, De Cristo Soares Alves A, Aguirre T, Pohlmann AR, Guterres SS, et al. 
Nasal drug delivery of anticancer drugs for the treatment of glioblastoma: Preclinical and clinical trials. Molecules. 2019;24(23).

33. Tan MSA, Parekh HS, Pandey P, Siskind DJ, Falconer JR. Nose-tobrain delivery of antipsychotics using nanotechnology: a review. Expert Opin Drug Deliv [Internet]. 2020;17(6):839-53. Available from: https://doi.org/10.1080/17425247.2 020.1762563

34. Bourganis V, Kammona O, Alexopoulos A, Kiparissides C. Recent advances in carrier mediated nose-to-brain delivery of pharmaceutics. Eur J Pharm Biopharm [Internet]. 2018;128:33762. Available from: https://doi.org/10.1016/j.ejpb.2018. 05.009

35. Pires PC, Santos AO. Nanosystems in nose-to-brain drug delivery: A review of non-clinical brain targeting studies. J Control Release [Internet]. 2018;270:89-100. Available from: http://dx.doi.org/10.1016/j.jconrel.2 017.11 .047

36. Cho K, Wang X, Nie S, Chen Z, Shin DM. Therapeutic nanoparticles for drug delivery in cancer. Clin Cancer Res. 2008;14(5):1310-6.

37. Nirale P, Paul A, Yadav KS. Nanoemulsions for targeting the neurodegenerative diseases: Alzheimer's, Parkinson's and Prion's. Life Sci [Internet]. 2020;245(December 2019):117394. Available from: https://doi.org/10.1016/j.lfs.2020.11 7394

38. Bonferoni MC, Rossi S, Sandri G, Ferrari F, Gavini E, Rassu G, et al. Nanoemulsions for "nose-to-brain" drug delivery. Pharmaceutics.
2019;11(2):1-17.

39. Kumar M, Misra A, Mishra AK, Mishra PP, Pathak K. Mucoadhesive nanoemulsion-based intranasal drug delivery system of olanzapine for brain targeting. J Drug Target. 2008;16(10):806-14.

40. Saka R, Chella N, Khan W. Development of Imatinib MesylateLoaded Liposomes for Nose to Brain Delivery: In Vitro and In Vivo Evaluation. AAPS PharmSciTech [Internet]. 2021;22(192). Available from: https://doi.org/10.1208/s12249-02102072-0

41. Shah P, Dubey P, Vyas B, Kaul A, Mishra AK, Chopra D, et al. Lamotrigine loaded PLGA nanoparticles intended for direct nose to brain delivery in epilepsy: pharmacokinetic, pharmacodynamic and scintigraphy study. Artif Cells, Nanomedicine, Biotechnol [Internet]. 2021;49(1):511-22. Available from: https://doi.org/10.1080/21691401.2 021.1939709

42. Bachhav SS, Dighe V, Mali N, Gogtay NJ, Thatte UM, Devarajan P V. Nose-to-Brain Delivery of Diazepam from an Intranasal AquaTriggered In-Situ (ATIS) Gelling Microemulsion: Monitoring Brain Uptake by Microdialysis. Eur J Drug Metab Pharmacokinet [Internet]. 2020;45(6):785-99. Available from: https://doi.org/10.1007/s13318-02000641-5

43. Youssef NAHA, Kassem AA, Farid RM, Ismail FA, EL-Massik MAE, Boraie NA. A novel nasal almotriptan loaded solid lipid nanoparticles in mucoadhesive in situ gel formulation for brain targeting: 
characterization and in vivo evaluation. Int $\mathbf{J}$ Pharm [Internet]. 2018;548(1):609-24. Available from:

https://doi.org/10.1016/j.ijpharm.20 18.07.014

44. Bayanati M, Khosroshahi AG, Alvandi M, Mahboobian MM. Fabrication of a Thermosensitive in Situ Gel Nanoemulsion for Nose to Brain Delivery of Temozolomide. J
Nanomater. 2021;2021.

45. Seju U, Kumar A, Sawant KK. Development and evaluation of olanzapine-loaded PLGA nanoparticles for nose-to-brain delivery: In vitro and in vivo studies. Acta Biomater [Internet]. 2011;7(12):4169-76. Available from:

http://dx.doi.org/10.1016/j.actbio.20 11.07.025 